\title{
Patient perception of inhaler use and its implications in inhaler management
}

\author{
Jin Sun Kim ${ }^{1}$, Hannah $\mathrm{Li}^{1}$, Nader Hashweh ${ }^{1}$, Salik Choudhary ${ }^{1}$, Sadashiv Santosh ${ }^{1}$, and \\ Edward Charbek ${ }^{2}$ \\ ${ }^{1}$ Saint Louis University School of Medicine \\ ${ }^{2}$ Saint Louis University
}

March 23, 2021

\begin{abstract}
Objectives: Literature has shown a high prevalence of poor inhaler technique amongst COPD patients throughout the past several decades. We aim to study the patients' perspective on the use of inhalers to understand how inhaler therapy can be better approached. Methods: COPD patients who were regularly using pressurized metered-dose inhaler(s) (pMDI) with or without spacers were recruited to complete a survey regarding their perception of inhaler use. Results: One hundred and one patients participated in the study. 91 (90.1\%) reported that they use their inhaler correctly and 80 (79.2\%) indicated that using their inhaler is easy. $35(34.7 \%)$ indicated that they prefer to consolidate all of their inhalers. When asked about previous training, $25(24.8 \%)$ reported that they have not been shown how to use inhalers before. Conclusions: Despite high prevalence of poor inhaler technique, COPD patients may not be aware of their poor technique- patients are confident in their use of inhalers and find its use easy. This discrepancy has not been discussed in literature before. Increasing patient awareness regarding poor inhaler technique and the importance of correct use of the device may be the key next step to address poor inhaler technique amongst COPD patients.
\end{abstract}

\section{Hosted file}

Manuscript IJCP.pdf available at https://authorea.com/users/403413/articles/514873-patientperception-of-inhaler-use-and-its-implications-in-inhaler-management 